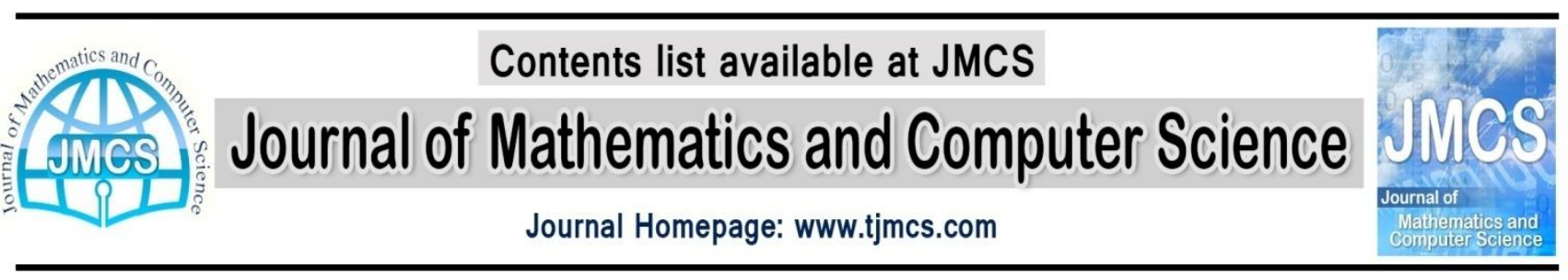

\title{
Fuzzy Ideals and Fuzzy Soft Ideals of BCH-Algebras
}

\author{
R. Moradian ${ }^{1}$, M. Hamidi ${ }^{2, *}$, A. Radfar ${ }^{2,+}$ \\ ${ }^{1}$ Department of Mathematics, Ilam University, Ilam, Iran \\ Rmoradian58@yahoo.com \\ ${ }^{2}$ Department of Mathematics, Payame Noor University, Tehran, Iran \\ *m.hamidi@pnu.ac.ir \\ +ateferadfar@yahoo.com
}

Article history:

Received November 2014

Accepted February 2015

Available online February 2015

\begin{abstract}
The purpose of this paper is the study of algebraic properties of soft sets in a $B C H$-algebras. In this regards we introduce and study soft ideals and idealistic soft $\mathrm{BCH}$-algebras.
\end{abstract}

Keywords: Soft set, (Idealistic) soft BCH-algebra, soft ideal

\section{Introduction}

In order to solve complicated problems in economics, engineering, environmental science, medical science, and social science, methods in classical mathematics are not always successfully used because various uncertainties are typical for these problems. Therefore, there has been a great deal of alternative research and applications in the literature concerning some special tools such as probability theory, fuzzy set theory [18, 19, 20], rough set theory [14, 15], vague set theory [3], and interval mathematics [4]. Although they are all useful approaches to describe uncertainty, each of these theories has its inherent difficulties, as mentioned by Molodtsov [13].Consequently, Molodtsov [13] proposed a completely new approach, called soft set theory, for modeling vagueness and uncertainty. Soft set theory has potential applications in many fields, including the smoothness of functions, game theory, operations research, Riemann integration, Perron integration, probability theory, and measurement theory. Most of these applications have already been demonstrated in Molodtsovs paper [13]. Currently, work on soft set theory is progressing rapidly. Maji et al. [11] investigated the applications of soft set theory to a decision making problem. Roy and Maji [16] proposed the concept of a fuzzy soft set and provided its properties and an application in decision making under an imprecise environment. Chen et al. [2] presented a definition for soft set parameterization reduction and showed an application in another decision making problem. Kong et al. [9] further studied the problem of the reduction of soft sets and fuzzy soft sets by introducing a definition for normal parameter reduction. Maji et al. [10] defined and studied several operations on soft sets, and Ali et al. [1] gave some new notions such as restricted intersection, restricted union, restricted 
difference, and extended intersection of soft sets. Jun [5] applied Molodtsovs notion of soft sets to the theory of BCK/BCI-algebras and introduced the notion of soft BCK/BCI-algebras and soft subalgebras and then investigated their basic properties. Jun and Park [6] dealt with the algebraic structure of BCK/BCI-algebras by applying soft set theory. They introduced the notion of soft ideals and idealistic soft BCK/BCI-algebras and gave several examples. Jun et al. [7] introduced the notion of soft p-ideals and p-idealistic soft BCI-algebras and investigated their basic properties. Using soft sets, they gave characterization of (fuzzy) p-ideals in BCI-algebras. Moreover, Jun et al. [8] applied a fuzzy soft set introduced by Maji et al. [12] as a generalization of the standard soft sets for dealing with several kinds of theories in BCK/BCI-algebras. They defined the notions of fuzzy soft BCK/BCI-algebras, (closed) fuzzy soft ideals, and fuzzy soft p-ideals, and investigated related properties. Yang et al. [17] introduced the concept of the interval-valued fuzzy soft set; they studied the algebraic properties of the concept and they analyzed a decision problem by using an interval-valued fuzzy soft set. In this paper, we deal with the algebraic structure of $B C H$-algebras by applying soft set theory. We discussed the algebraic properties of soft sets in $B C H$-algebras and introduced the notion of soft ideals and idealistic soft $\mathrm{BCH}$-algebras. For there more we investigated relation between soft $B C H$-algebra and idealistic soft $B C H$-algebras. In follows we established the intersection, union, "AND" operation and "OR" operation of soft ideals and idealistic soft $\mathrm{BCH}$-algebras.

\section{Preliminaries}

In this section we gather some basic definitions and results on $\mathrm{BCH}$-algebras and soft sets which we need to extending our paper. Recall that a $B C H$-algebra is an algebra $(X, *, 0)$ of type $(2,0)$ satisfying the following axioms:

(BCH1) $x * x=0$,

$(\mathrm{BCH} 2)(x * y) * z=(x * z) * y$,

(BCH4) $x * y=0$ and $y * x=0$ imply $x=y$.

for every $x, y, z \in X$. For any $B C H$-algebra $X$, the relation $\leq$ defined by $x \leq y$ if and only if $x * y=0$ is a partial order on $X$. In any $B C H$-algebra $X$, the following hold:

(u1) $x * 0=x$

(u2) $0 *(x * y)=(0 * x) *(0 * y)$,

for all $x, y \in X$. A nonempty subset $S$ of a $B C H$-algebra $X$ is said to be a subalgebra of $X$ if $x * y \in S$, whenever $x, y \in S$. A nonempty subset $A$ of a $B C H$-algebra $X$ is called an ideal, denoted by $A \unlhd X$, if it satisfies:

(I1) $0 \in A$,

(I2) $x * y \in A$ and $y \in A$ imply $x \in A$ for all $x, y, z \in X$.

Note that an ideal of a $B C H$-algebra $X$ is a subalgebra of $X$. Let $U$ be an initial universe set, $E$ be a set of parameters, $P(U)$ denotes the power set of $U$ and $A \subset E$. Then we have the following definitions:

Definition 2.1. [13] A pair $(p, A)$ is called a soft set over $U$, where $p$ is a mapping given by

$$
p: A \rightarrow P(U) \text {. }
$$

In other words, a soft set over $U$ is a parameterized family of subsets of the universe $U$. For $a \in A, p(a)$ may be considered as the set of $a$-approximate elements of the soft set $(p, A)$. Clearly, a soft set is not a set.

Definition 2.2. [11]Let $(p, A)$ and $(q, B)$ be two soft sets over a common universe $U$.

(i) The intersection of $(p, A)$ and $(q, B)$ is defined to be the soft set $(r, C)$ satisfying the following conditions:

(1) $C=A \cap B$,

(2) $(\forall e \in C)(r(e)=p(e)$ or $q(e)$, (as both are same set)).

In this case, we write $(p, A) \widetilde{\cap}(q, B)=(r, C)$. 
(ii) Let $\left\{\left(p_{i}, A_{i}\right) \mid i \in I\right\}$ be a family of soft sets over a common universe $U$. The intersection $\bigcap_{i \in I}\left(p_{i}, A_{i}\right)$ is defined to be the soft set $(r, C)$ satisfying the following conditions:

(1) $C=\bigcap_{i \in I} A_{i}$

(2) $(\forall e \in C)\left(r(e)=p_{i}(e)\right.$ or $p_{-} j(e),(i, j \in I)$, (as both are same set)).

In this case, we write $\widetilde{\cap}_{i \in I}\left(p_{i}, A_{i}\right)=(\mathrm{r}, \mathrm{C})$.

Definition 2.3. [11] Let $(p, A)$ and $(q, B)$ be two soft sets over a common universe $U$.

(i) The union of $(p, A)$ and $(q, B)$ is defined to be the soft set $(r, C)$ satisfying the following conditions:

(1) $C=A \cup B$,

(2) for all $e \in C$,

$$
r(e)= \begin{cases}p(e) & , \text { if } e \in A \backslash B \\ q(e) & , \text { if } e \in B \backslash A \\ p(e) \cup q(e) & , \text { if } e \in A \cap B\end{cases}
$$

In this case, we write $(p, A)) \widetilde{U}(q, B)=(r, C)$.

(ii) Let $\left\{\left\{\left(p_{i}, A_{i}\right) \mid i \in I\right\}\right.$ be a family of soft sets over a common universe $U$. The union $\bigcup_{i \in I}\left(p_{i}, A_{i}\right)$ is defined to be the soft set $(r, C)$ satisfying the following conditions:

(1) $C=\bigcup_{i \in I} A_{i}$,

(2) for all $e \in C$,

In this case, we write $\underset{i \in I}{\widetilde{U}}\left(p_{i}, A_{i}\right)=(r, C)$.

$$
r(e)=\left\{\begin{array}{cl}
p_{i}(e) & \text {, if } e \in A_{i} \backslash \bigcup_{i \neq j} A_{j}, \\
\bigcup_{i \in I} p_{i}(e) & \text {, if } e \in \bigcap_{i \in I} A_{i}
\end{array}\right.
$$

Definition 2.4. [11] Let $(p, A)$ and $(q, B)$ be two soft sets over a common universe $U$. Then:

i) " $(p, A) \operatorname{AND}(q, B)$ "denoted by $(p, A)) \tilde{\Lambda}(q, B)$ is defined by $(p, A) \tilde{\Lambda}(q, B)=(r, A \times B)$, where $r(\alpha, \beta)=p(\alpha) \cap q(\beta)$ for all $(\alpha, \beta) \in A \times B$.

ii) " $(p, A)$ OR $(q, B)$ "denoted by $(p, A) \widetilde{\mathrm{V}}(q, B)$ is defined by $(p, A) \widetilde{\mathrm{V}}(q, B)=(r, A \times B)$, where $r(\alpha, \beta)=p(\alpha) \cup q(\beta)$ for all $(\alpha, \beta) \in A \times B$.

Definition 2.5. [11] Let $(p, A)$ and $(q, B)$ be two soft sets over a common universe $U$. We say that $(p, A)$ is a soft subset of $(q, B)$, denoted by $(p, A) \widetilde{\subset}(q, B)$, if it satisfies:

(i) $A \subset B$,

(ii) For every $a \in A, p(a)$ and $q(a)$ are identical approximations.

\section{Soft Ideal}

In what follow let $X$ and $A$ be a $B C H$-algebra and a non empty set, respectively, and $R$ will refer to an arbitrary binary relation between an element of $A$ and an element of $X$, that is, $R$ is a subset of $A \times X$ without otherwise specified. A set-values function $p: A \rightarrow p(X)$ can be defined as $p(x)=\{y \in$ $X \mid(x, y) \in R\}$ for all $x \in A$. The pair $(p, A)$ is then a soft set over $X$.

Definition 3.1. A non empty subset $S$ of a $B C H$-algebra $(X, *, 0)$ is called a subalgebra if $x * y \in S$, for all $x, y \in S$.

Definition 3.2. Let $S$ be a subalgebra of $X$. A subset $I$ of $X$ is called an ideal of $X$ related to $S$ (briefly, $S$ ideal of $X$ ), denoted by $I \triangleleft S$, if it satisfies: 
(i) $0 \in I$,

(ii) $(\forall x \in S)(\forall y \in I)(x * y \in I \Rightarrow x \in I)$.

Note that if $S$ is a subalgebra of $X$ and $I$ is a subset of $X$ that contains $S$ then $I$ is an $S$-ideal of $X$. Obviously, every ideal of $X$ is an $S$-ideal of $X$ for every subalgebra $S$ of $X$, but the converse is not true in general as seen the following example.

Example 3.3. Let $X=\{0,1,2,3,4\}$ be a $B C H$-algebra with the following Cayley table:

\begin{tabular}{llllll}
$*$ & 0 & 1 & 2 & 3 & 4 \\
\hline$\oint$ & 0 & 0 & 0 & 0 & 4 \\
1 & 1 & 0 & 0 & 1 & 4 \\
2 & 2 & 2 & 0 & 0 & 4 \\
3 & 3 & 3 & 0 & 4 \\
4 & 4 & 4 & 4 & 4 & 0
\end{tabular}

Then $S=\{0,1\}$ is a subalgebra of $X$ and $I=\{0,1,3\} \triangleleft S$, but $I$ is not an ideal of $X$ because $2 * 3=$ $0 \in I$ and $2 \notin I$.

Definition 3.4. Let $(p, A)$ be a soft set over $X$. Then $(p, A)$ is called a soft $B C H$ algebra over $X$ if $p(x)$ is a subalgebra of $X$ for all $x \in A$.

Definition 3.5. Let $(p, A)$ be a soft $B C H$-algebra over $X$. A soft set $(q, I)$ over $X$ is called a soft ideal of $(p$, $A)$, denoted by $(q, I) \widetilde{\triangleleft}(p, A)$, if it satisfies:

(i) $I \subset A$,

(ii) $(\forall x \in A)(q(x) \triangleleft p(x))$.

Let us illustrate this definition using the following examples.

Example 3.6. Let $X=\{0, a, b, c\}$ be a $B C H$-algebra with the following cayley table:

$$
\begin{array}{l|llll}
* & 0 & a & b & c \\
\hline 0 & 0 & a & b & c \\
a & a & 0 & c & b \\
b & b & c & 0 & a \\
c & c & b & a & 0
\end{array}
$$

Let $(p, A)$ be a soft set over $X$, where $A=X$ and $p: A \rightarrow p(X)$ is a set-valued function defined by $p(x)=$ $\{y \in X \mid x *(y * x) \in\{0, x\}\}$ for all $x \in A$. Then $\mathrm{p}(0)=\{0\}, p(a)=\{0, a\}, p(b)=\{0, b\}$ and $p(c)=\{0, c\}$ are subalgebra of $X$. Then $(p, A)$ is a soft $B C H$-algebra over $X$.

(1) Let $(q, I)$ be a soft set over $X$, where $I=\{a, b\}$ and $q: I \rightarrow p(X)$ is a set-valued function defined by $q(x)=\{y \in X \mid y *(y * x) \in\{0, x, c\}\}$ for all $x \in I$. Then $q(a)=\{0, a, b, c\} \triangleleft\{0, a\}=p(a), q(b)=\{0, a, b, c\} \triangleleft p(b)=\{0, b\}$. Hence $(q, I)$ is a soft ideal of $(p, A)$.

(2) For $I=\{a, b\}$, let $r: I \rightarrow p(X)$ be a set-valued function defined by $r(x)=\{0\} \cup\{y \in X \mid y \leq$ $x\}$ for all $x \in I$. Then $r(a)=\{0, a\} \triangleleft\{0, a\}=p(a)$ and $r(b)=\{0, b\} \triangleleft\{0, b\}=p(b)$. Therefore, $(r, I)$ is a soft ideal of $(p, A)$.

Theorem 3.7. Let $(p, A)$ be a soft BCH-algebra over $X$. For any soft sets $\left(q_{1}, I_{1}\right)$ and $\left(q_{2}, I_{2}\right)$ over $X$ where $I_{1} \cap I_{2} \neq \emptyset$, we have

$$
\left(q_{1}, I_{1}\right) \widetilde{\triangleleft}(p, A),\left(q_{2}, I_{2}\right) \widetilde{\triangleleft}(p, A) \Rightarrow\left(q_{1}, I_{1}\right) \widetilde{\cap}\left(q_{2}, I_{2}\right) \widetilde{\triangleleft}(p, A)
$$

Proof. Using Definition 2.2, we can write

$$
\left(q_{1}, I_{1}\right) \widetilde{\cap}\left(q_{2}, I_{2}\right)=(q, I)
$$


where $I=I_{1} \cap I_{2}$ and $q(x)=q_{1}(x)$ or $q_{2}(x)$ for all $x \in I$. Obviously, $I \subset A$ and $q: I \rightarrow p(X)$ is a mapping. Hence $(q, I)$ is a soft set over $X$. Since $\left(q_{1}, I_{1}\right) \widetilde{\triangleleft}(p, A)$ and $\left(q_{2}, I_{2}\right) \widetilde{\triangleleft}(p, A)$, we know that $q(x)=q_{1}(x) \triangleleft p(x)$ or $q(x)=q_{2}(x) \triangleleft p(x)$ for all $x \in I$. Therefore, $\left(q_{1}, I_{1}\right) \widetilde{\cap}\left(q_{2}, I_{2}\right)=$ $(q, I) \widetilde{\triangleleft}(p, A)$.

Corollary 3.8. Let $(p, A)$ be a soft BCH-algebra over X. For any soft sets $(q, I)$ and $(r, I)$ over $X$, we have $(q, I) \widetilde{\triangleleft}(p, A),(r, I) \widetilde{\triangleleft}(p, A) \Rightarrow(q, I) \widetilde{\cap}(r, I) \widetilde{\triangleleft}(p, A)$

Theorem 3.9. Let $(p, A)$ be a soft BCH-algebra over X. For any soft sets $(q, I)$ and $(r, J)$ over $X$ in which $I$ and $J$ are disjoint, we have

$$
(q, I) \widetilde{\triangleleft}(p, A),(r, J) \widetilde{\triangleleft}(p, A) \Rightarrow(q, I) \widetilde{\cup}(r, J) \widetilde{\triangleleft}(p, A)
$$

Proof. Assume that $(q, I) \widetilde{\triangleleft}(p, A)$ and $(r, J) \widetilde{\triangleleft}(p, A)$. By means of definition 2.3 , we can write $(q, I) \widetilde{\cup}(r, J)=(s, K)$ where $K=I \cup J$ and for every $x \in K$,

$$
s(x)=\left\{\begin{array}{cl}
q(x) & \text { if } x \in I \backslash J \\
r(x) & \text { if } x \in J \backslash J \\
q(x) \cup r(x) & \text { if } x \in I \cap J
\end{array}\right.
$$

Since $I \cap J=\phi$, either $x \in I \backslash J$ or $x \in J \backslash I$ for all $x \in K$.

If $x \in I \backslash J$, then $s(x)=q(x) \triangleleft p(x)$. If $x \in J \backslash I$, then $s(x)=r(x) \triangleleft p(x)$. Thus $s(x) \triangleleft p(x)$ for all $x \in K$, and so $(q, I) \widetilde{\cap}(r, J)=(s, K) \widetilde{\triangleleft}(p, A)$.

Note that if $I$ and $J$ are not disjoint in Theorem 3.9, then Theorem 3.9, is not true in general seen in the following example.

Example 3.10. Let $X=\{0,1,2,3\}$ in which $*$ is defined by:

Then $(X, *, 0)$ is a $B C H$-algebra.

$$
\begin{array}{l|llll}
* & 0 & 1 & 2 & 3 \\
\hline 0 & 0 & 0 & 0 & 0 \\
1 & 1 & 0 & 3 & 3 \\
2 & 2 & 0 & 0 & 2 \\
3 & 3 & 0 & 0 & 0
\end{array}
$$

Let $(p, A)$ be a soft set over $X$, where $A=X$ and $p: A \rightarrow p(X)$ is a set-valued function defined by $p(x)=\{y \in X \mid y *(y * x) \in\{0,3\}\}$ for all $x \in A$. Then $p(0)=p(3)=X, p(1)=\{0,3\}$ and $p(2)=\{0,1,3\}$ which are subalgebras of $X$. Hence $(p, A)$ is a soft $B C H$-algebra over $X$.

Let $I=\{0,2\}$ and $q: I \rightarrow p(X)$ is a set-valued function defined by $q(x)=\{y \in X \mid y *(y * x) \in$ $\{0,2\}\}$ for all $x \in I$. Then $q(0)=X \triangleleft X=p(0)$ and $q(2)=\{0,2\} \triangleleft\{0,1,3\}=p(2)$, and so $(q$, $I)$ is a soft ideal of $(p, A)$.

Let $J=\{2,3\}$ and $r: J \rightarrow p(X)$ is a set-valued function defined by $r(x)=\{y \in X \mid y * x=$ $0\} \backslash\{x\}$ for all $x \in J$. Then $r(2)=\{0,3\} \triangleleft p(2)=\{0,1,3\}$ and $r(3)=\{0\} \triangleleft X=p(3)$, andso $(r$, $J)$ is a soft ideal of $(p, A)$. Then $(s, U)=(q, I) \sim U(r, J)$ is not a soft ideal of $(p, A)$, since $1 * 2=3 \in$ $s(2)$ and $2 \in s(2)$ but $1 \notin s(2) s(2)=r(2) \cup q(2)=\{0,2,3\}$, we have is not $p(2)$-ideal.

\section{Idealistic soft $\mathrm{BCH}$-algebra}

Definition 4.1. Let $(p, A)$ be a soft set over $X$. Then $(p, A)$ is called an idealist soft $B C H$-algebra over $X$ if $p(x)$ is an ideal of $X$ for all $x \in A$.

Example 4.2. Let $X=\{0,1,2,3,4\}$ in which $*$ is defined by:

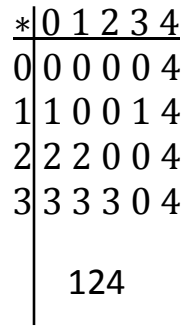




\section{0}

Routine calculations give that $(X, *, 0)$ is a $B C H$-algebra.

Let $A=\{0,1,2\}$ and let $p: A \rightarrow p(X)$ be a set-valued function defined by $p(x)=\{y \in X \mid y * x=$ $0\}$ for all $x \in A$. Then $p(0)=\{0\}, p(1)=\{0,1\}$ and $p(2)=\{0,1,2\}$, which areideals of $X$. Hence $(p, A)$ is an idealistic soft $B C H$-algebra over $X$.

Proposition 4.3. Let $(p, A)$ and $(q, B)$ be soft sets over $X$ where $B \subseteq A \subseteq X$. If $(p, A)$ is an idealistic soft $B C H$-algebra over $X$, then so is $(q, B)$.

Proof. The proof is straightforward.

Note that example 3.6[18] show that the converse of proposition 4.3 is not true in general.

Theorem 4.4. Let $(p, A)$ and $(q, B)$ be two idealistic soft $B C H$-algebra over $X$. If $A \cap B \neq \emptyset$, then the intersection $(p, A) \sqcap(q, B)$ is an idealistic soft BCH-algebra over $X$.

Proof. The proof is similar to the proof of Theorem 3.7.

Corollary 4.5. Let $(p, A)$ and $(q, A)$ be two idealistic soft BCH-algebra over X. Then their intersection $(p, A) \sqcap(q, A)$ is an idealistic soft BCH-algebra over X.

Theorem 4.6. Let $(p, A)$ and $(q, B)$ be two idealistic soft $B C H$-algebra over $X$. If $A$ and $B$ are disjoint, then the union $(p, A) \sqcup(q, B)$ is an idealistic soft $B C H$-algebra.

Proof. The proof is similar to the proof of Theorem 3.9.

Theorem 4.7. Let $(p, A)$ and $(q, B)$ be two idealistic soft BCH-algebra over $X$. Then $(p, A) \widetilde{\Lambda}(q, B)$ is an idealistic soft $B C H$-algebra over $X$.

Proof. By use of Definition 2.4, we know that $(p, A) \tilde{\wedge}(q, B)=(r, A \times B)$, where $r(x, y)=p(x) \cap$ $q(y)$ for all $(x, y) \in A \times B$. Since $p(x)$ and $q(y)$ are ideals of $X$, the intersection $p(x) \cap q(y)$ isalso an ideal of $X$. Hence $r(x, y)$ is an ideal of $X$ for all $(x, y) \in A \times B$. Therefore, $(p, A) \tilde{\Lambda}(q, B)=(r, A \times B)$ is an idealistic soft $B C H$-algebra over $X$.

Definition 4.8. An idealistic soft $B C H$-algebra $(p, A)$ is said to be trivial (resp., whole) if $p(x)=\{0\}$ (resp., $p(x)=X$ ) for all $x \in A$.

Example 4.9. Let $(X, *, 0)$ be a $B C H$-algebra which $X=\{0,1,2,3,4\}$ and $*$ is defined by:

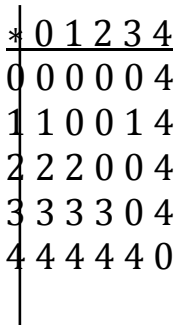

Suppose that $A=\{0,1,2,3\}$ and $p: A \rightarrow p(X)$ be a set-valued function defined by $p(x)=\{y \in$ $X \mid(y * x) * x=y * x\}$, Then $p(x)=X$ for all $x \in A$, and so $(p, A)$ is a whole idealistic soft $B C H$ algebra over $X$.

Lemma 4.10. Let $f: X \rightarrow Y$ be a mapping of $B C H$-algebras.

(i) For a soft set $(p, A)$ over $X,(f(p), A)$ is a soft set over $Y$ where $f(p): A \rightarrow p(Y)$ is defined by $f(p)(x)=f(p(x))$ for all $x \in A$. 
(ii) For a soft set $(q, B)$ over $Y,\left(f^{-1}(q), B\right)$ is a soft set over $X$, where $f^{-1}(q): B \rightarrow P(X)$ is defined by $f^{-1}(q)(b)=\cup_{y \in q(b)} f^{-1}(y)$ for all $b \in B$.

Proof. The proof is straightforward.

Lemma 4.11. Let $f: X \rightarrow Y$ be an onto homomorphism of $B C H$-algebras.

(i) If $(p, A)$ is an idealistic soft BCH-algebras over $X$. Then $(f(p), A)$ is an idealistic soft BCH-algebras over $Y$.

(ii) If $(q, B)$ is an idealistic oft BCH-algebra over $Y$, then $\left(f^{-1}(q), B\right)$ is an idealistic soft BCH-algebra over $X$.

Proof. (i) For every $x \in A$, we have $f(p)(x) \in f(p(x))$ is an ideal of $Y$ since $p(x)$ is an ideal of $X$ and its onto homomorphic image is also an ideal of $Y$. Therefore, $(f(p), A)$ is an idealistic soft $B C H$-algebras over $Y$.

(ii) First we prove that if $B$ be an ideal of $Y$, then $f^{-1}(B)$ is an ideal of $X$. Obviously

we have $0 \in f^{-1}(B)$. Now, let $x, y \in X$ be such that $x * y \in f^{-1}(B)$ and $y \in f^{-1}(B)$, so we have $f(x * y)=f(x) * f(y) \in B$ and $f(y) \in B$. Since $B$ is an ideal of $Y$, we have $f(x * y)=f(x) *$ $f(y) \in B$ and so $x * y \in f^{-1}(B)$. Thus $f^{-1}(B)$ is an ideal of $X$. Now, for every $b \in B$, since $q(b)$ is an ideal of $Y$, we have $f^{-1}(q)(b)=\cap f^{-1}(y)$ is an ideal of $X$. Thus, $\left(f^{-1}(q), B\right)$ is an idealistic soft $B C H$ algebra over $X$.

Theorem 4.12. Let $f: X \rightarrow Y$ be an onto homomorphism of BCH-algebras and let $(p, A)$ bean idealistic soft $B C H$-algebras over $X$. Then:

(i) Let $p(x)=$ kerf for all $x \in A$. Then $(f(p), A)$ is the trivial idealistic soft BCH-algebraover $Y$.

(ii) Let $(p, A)$ is whole. Then $(f(p), A)$ is the whole idealistic soft BCH-algebra over $Y$.

Proof. (i) Assume that $p(x)=\operatorname{ker}(f)$ for all $x \in A$. Then $f(p)(x)=f(p(x))=\left\{0_{Y}\right\}$ for all $x \in A$. Hence, by Definition 4.8 and Lemma 4.11, $(f(p), A)$ is the trivial idealistic soft BCH-algebra over $Y$.

(ii) Suppose that $(p, A)$ is whole. Then $p(x)=X$ for all $x \in A$ and so $f(p)(x)=f(p(x))=f(X)=$ $Y$ for all $x \in A$. It follows from Definition 4.8 and Lemma 4.11, that $(f(p), A)$ is the whole idealistic soft $B C H$-algebra over $Y$.

\section{Fuzzy ideal and fuzzy soft ideal of $B C H$-algebras}

Definition 5.1. A fuzzy subset $\mu$ of a $B C H$-algebra $X$ is said to be a fuzzy ideal of $X$ if it satisfies:

(i) $\mu(0) \geq \mu(x)$ for all $x \in X$,

(ii) $\mu(x) \geq \min \{\mu(x * y), \mu(y)\}$ for all $x, y, z \in X$.

Definition 5.2. Let $X$ be a $B C H$-algebra and $F(X)$ be the set of fuzzy set over $X$. A pair $(p, A)$ is called a fuzzy soft set over $B C H$-algebra $X$, where $p$ is a mapping given by:

$$
p: A \rightarrow F(X)
$$

In other word, for every $a \in A, p_{a}: X \rightarrow[0,1]$ is a fuzzy set over $X$. Note that for every fuzzy set $\mu$, the set $\mu_{t}=\{x \in X \mid \mu(x) \geq t\}$ is called $t$-level relation over BCH-algebra $X$.

Definition 5.3. A fuzzy soft set $(p, A)$ over $B C H$-algebra $X$ is called fuzzy soft ideal, if for every $a \in A$, $p_{a} \in F(X)$ be a fuzzy ideal of $X$.

Theorem 5.4. Let $(p, A)$ be a fuzzy soft set over BCH-algebra and a $\in A$, then $p_{a} \in F(X)$ is a fuzzy ideal if and only if $\left(p_{a}\right)_{t} \neq \varnothing$ is an ideal of $B C H$-algebra $X$.

Proof. Let $p_{a} \in F(X)$ be a fuzzy ideal, we must prove that $\left(p_{a}\right)_{t} \neq \varnothing$ is an ideal of BCH-algebra. Since $p_{a}(0) \geq p_{a}(x)$, obviously we have $0 \in\left(p_{a}\right)_{t}$. Now, let $x, y \in X$ be such that $(x * y) \in\left(p_{a}\right)_{t}, y \in$ $\left(p_{a}\right)_{t}$, then $p_{a}(x * y) \geq t, p_{a}(y) \geq t$. So we have:

$$
p_{a}(x) \geq \min \left\{p_{a}(x * y), p_{a}(y)\right\} \geq t \text {. }
$$

Hence $x \in\left(p_{a}\right)_{t}$. Therefore $\left(p_{a}\right)_{t}$ is an ideal of BCH-algebra. 
Conversely, suppose that $\left(p_{a}\right)_{t} \neq \varnothing$ is an ideal of $X$, we must prove that $p_{a}$ is a fuzzy ideal of $X$. For any $x \in X$, since $x \in\left(p_{a}\right)_{p_{a}(x)} \neq \varnothing$ is an ideal and so $0 \in\left(p_{a}\right)_{p_{a}(x)}$, that is $p_{a}(0) \geq p_{a}(x)$.

Now, for any $x, y \in X$, we let

$$
t=\min \left\{p_{a}(x * y), p_{a}(y)\right\}
$$

it followes that $(x * y) \in\left(p_{a}\right)_{t}$ and $y \in\left(p_{a}\right)_{t}$. Since $\left(p_{a}\right)_{t} \neq \varnothing$ is an ideal of BCH-algebra, we have $x \in\left(p_{a}\right)_{t}$. Therefore,

This complete the proof.

$$
p_{a}(x) \geq t=\min \left\{p_{a}(x * y), p_{a}(y)\right\}
$$

We denote the set of soft ideal, fuzzy ideal and fuzzy soft ideal that constructed over BCH-algebra X by $S I(x), F I(x)$ and $F S I(x)$, respectively.

Definition 5.5. Let $X$ be a $B C H$-algebra and $(p, A)$ be a soft $B C H$-algebra over $X$, we say that $(p, A)$ satisfies the maximal condition, if each nonempty subset of $S I(p, A)$ contains least one maximal member with respect to the set theoretical inclusion $\subseteq$ and $(p, A)$ satisfies the ascending chain condition, abbreviated by ACC, if there does not exist an infinite property ascending chain $\left(q_{1}, I_{1}\right) \subseteq\left(q_{2}, I_{2}\right) \subseteq \cdots$ in $\operatorname{SI}(p, A)$. In an entirely analogous way the minimal condition and the descending chain condition (abbreviated by DCC) are defined.

Theorem 5.6. Let $X$ be a BCH-algebra and $(p, A)$ be a soft $B C H$-algebra over $X$, then

(i) $(p, A)$ satisfies the maximal condition if and only if $(p, A)$ satisfies $A C C$.

(ii) $(p, A)$ satisfies the minimal condition if and only if $(p, A)$ satisfies DCC.

Proof. (i) Suppose $(p, A)$ satisfies the maximal condition and $\left(q_{1}, I_{1}\right) \subseteq\left(q_{2}, I_{2}\right) \subseteq \cdots$ is a ascending chain in $\operatorname{SI}(X)$. Then the set $\left\{\left(q_{i}, I_{i}\right): i=1,2, \ldots\right\}$ has maximal member

$\left(q_{n}, I_{n}\right)$, so $\left(q_{i}, I_{i}\right)=\left(q_{n}, I_{n}\right)$ for all $i \geq n$, this says $(p, A)$ satisfies ACC.

Conversely, suppose that ( $p, A)$ satisfies $\mathrm{ACC}$ and $E$ is any nonempty subset of $\operatorname{SI}(X)$.

If $E$ has no maximal member, then each member of $E$ precede another member of $E$,

which permits the construction of an infinite chain $\left(q_{1}, I_{1}\right) \subseteq\left(q_{2}, I_{2}\right) \subseteq \cdots$ in $E$, where $\left(q_{i}, I_{i}\right) \neq$ $\left(q_{j}, I_{j}\right)$ whenever $i \neq j$, a contradiction. Hence $(p, A)$ satisfies the maximal condition. Likewise for $(i i)$, the reader should supply the details.

\section{Conclusions and further research}

Soft sets are deeply related to fuzzy sets and rough sets. we applied soft sets to BCH-algebra. we discussed the algebraic properties of soft sets in $B C H$-algebras. We introduced the notion of soft ideals and idealistic soft $B C H$-algebras, and gave several example. we investigated relation between soft $B C H$ algebra and idealistic soft $B C H$-algebra. we established the union, intersection, "AND" operation, and "OR" operation of soft ideals and idealistic soft $B C H$-algebra. Based on these results, we will apply soft sets to ideals on hyper $B C K$-algebra, and investigated relation between fuzzy type of ideals.

\section{References}

[1] M.I. Ali, F. Feng, X. Liu,W.K. Min, “On some new operations in soft set theory”, Comput. Math. Appl. 57 (9) (2009) 1547-1553.

[2] D. Chen, E.C.C. Tsang, D.S. Yeung, X.Wang, "The parameter reduction of soft sets and its algorithm",Comput. Math. Appl. 56 (12) (2008) 3029-3037.

[3] W.L. Gau, D.J. Buehrer, "Vague sets", IEEE Trans. Syst. Man Cybern. 23 (2) (1993) 610-614.

[4] M.B. Gorzalzany, "A method of inference in approximate reasoning based on interval-valued fuzzy sets", FuzzySets and Systems 21 (1987) 117.

[5] Y.B. Jun, "Soft BCK/BCI-algebras", Comput. Math. Appl. 56 (2008) 1408-1413.

[6] Y.B. Jun, C.H. Park, "Applications of soft sets in ideal theory of BCK/BCI-algebras", Inf. Sci. 178 (2008)2466-2475. 
[7] Y.B. Jun, K.J. Lee, J. Zhan, “Soft p-ideals of soft BCI-algebras”, Comput. Math. Appl. 58 (2009) 2060-2068.

[8] Y. B. Jun, "Soft BCK/BCI algebras", Computers and Mathematics with Applications 56 (5) (2008) 1408-1413.

[9] Y.B. Jun, K.J. Lee, C.H. Park, "Fuzzy soft set theory applied to BCK/BCI-algebras", Comput. Math. Appl.59 (2010) 3180-3192.

[10] Z. Kong, L. Gao, L.Wang, S. Li, "The parameter reduction of soft sets and its algorithm", Comput. Math.Appl. 56 (12) (2008) 3029-3037.

[11] P.K. Maji, R. Biswas, A.R. Roy, "Soft set theory", Comput. Math. Appl. 45 (2003) 555-562.

[12] P.K. Maji, A.R. Roy, R. Biswas, "An application of soft sets in a decision making problem", Comput. Math.Appl. 44 (2002) 1077-1083.

[13] P.K. Maji, R. Biswas, A.R. Roy, "Fuzzy soft sets", J. Fuzzy Math. 9 (3) (2001) 589-602.

[14] D. Molodtsov, "Soft set theory rst results", Comput. Math. Appl. 37 (1999) 1931.

[15] Z. Pawlak, "Rough sets", Int. J. Inf. Comput. Sci. 11 (1982) 341-356.

[16] Z. Pawlak, A. Skowron, "Rudiments of rough sets”, Inf. Sci. 177 (2007) 327.

[17] A.R. Roy, P.K. Maji, "A fuzzy soft set theoretic approach to decision making problems", J. Comput. Appl.Math. 203 (2007) 412-418.

[18] X.B. Yang, T.Y. Lin, J.Y. Yang, Y. Li, D.J. Yu, "Combination of interval-valued fuzzy set and soft set", Comput. Math. Appl. 58 (2009) 521-527.

[19] L.A. Zadeh, "Fuzzy sets", Inf. Control 8 (1965) 338-353.

[20] L.A. Zadeh, "Toward a generalized theory of uncertainty (GTU)-an outline", Inf. Sci. 172 (2005) 140. 